\begin{tabular}{|r|l|}
\hline \multicolumn{2}{|c|}{ Statistica Sinica Preprint No: SS-2020-0421 } \\
\hline Title & $\begin{array}{l}\text { Robust Feature Screening via Distance Correlation for } \\
\text { Ultrahigh Dimensional Data With Responses Missing at } \\
\text { Random }\end{array}$ \\
\hline Manuscript ID & SS-2020-0421 \\
\hline URL & http://www.stat.sinica.edu.tw/statistica/ \\
\hline DOI & $10.5705 /$ ss.202020.0421 \\
\hline Complete List of Authors & $\begin{array}{l}\text { Linli Xia and } \\
\text { Niansheng Tang }\end{array}$ \\
\hline Corresponding Author & Niansheng Tang \\
\hline E-mail & nstang@ynu.edu.cn \\
\hline Notice: Accepted version subject to English editing. \\
\hline
\end{tabular}


Statistica Sinica

\title{
Feature screening via distance correlation for ultrahigh dimensional data with responses missing at random
}

\author{
Linli Xia and Niansheng Tang \\ Yunnan Key Laboratory of Statistical Modeling and Data Analysis \\ Yunnan University, Kunming 650500, China
}

Abstract: This paper studies the feature screening problem for ultrahigh dimensional data with responses missing at random. A two-step procedure is proposed to screen important features. The first step is to screen the significant covariates associated with missing indicators via the fused mean-variance filter. The second step is to screen the important predictors associated with response by fusing distance correlation and nonparametric imputation technique. The proposed feature screening procedure has the following merits: (i) it is model free because it does not depend on a special model structure and distribution assumption; (ii) it avoids resampling on the conditional function of missing value because the kernel smoothing technique is adopted to implement nonparametric conditional mean imputation; (iii) it is not sensitive to the misspecification of propensity score function because of without imposing a special model on respondent probability. Under some regularity conditions, the sure screening property is shown. A modified maximum ratio criterion is proposed to select the tuning parameter. Simulation studies are conducted 
to investigate the finite sample performance of the proposed feature screening procedure.

An example is used to illustrate the proposed methodologies.

Key words and phrases: Distance correlation, Missing at random, Nonparametric imputation, Sure screening property, Ultrahigh dimensional data.

\section{Introduction}

Ultrahigh-dimensional data are often encountered in many fields of modern scientific research, such as signal processing, biomedical imaging and functional magnetic resonance imaging and finance, where the number of candidate predictors $p$ may increase at an exponential rate of the sample size $n$, while only a small number of predictors contribute to the response when there is the sparsity among the candidate predictors. Under the "larger $p$ smaller $n$ " data framework, various penalized variable selection procedures have been developed to reduce dimensionality from high to a small number that is below the sample size by effectively distinguishing important predictors. For example, see LASSO (Tibshirani, 1996), SCAD (Fan and Li, 2001), Adaptive LASSO (Zou, 2006), and Minimax Concave Penalty (Zhang, 2010). The aforementioned penalized variable selection methods may not perform well for ultrahigh-dimensional data due to the simultaneous challenges of computational expediency, statistical accuracy and algorithmic stability (Fan, Samworth and Wu, 2009).

To accommodate these challenges, various feature screening procedures have 
been developed for ultrahigh-dimensional data. For instance, Fan and Lv (2008) proposed a sure independence screening (SIS) and iterated sure independence screening (ISIS) procedure to select active predictors by ranking the marginal correlations for a linear model with Gaussian covariates and responses. Fan and Song (2010) presented a more general version of the independent learning by ranking maximum marginal likelihood estimates or maximum marginal likelihood itself in generalized linear models. Fan et al. (2011) developed a nonparametric independence screening (NIS) method for sparse ultrahigh dimensional additive models. Chang et al. (2013) presented a marginal-empirical-likelihood based independence feature screening procedure for linear regression models. The aforementioned methods depend on the considered models. To this end, some model-free feature screening approaches have been proposed in recent years. For example, see the sure independent ranking and screening (Zhu et al., 2011), robust-ranking-correlationbased screening (Li et al., 2012), distance-correlation-based screening (DC-SIS) (Li, Zhong and Zhu, 2012; Zhong et al., 2016), mean-variance sure independence screening (MV-SIS) (Cui, Li and Zhong, 2015), fused Kolmogorov filter screening (Mai and Zou, 2015), conditional quantile sure independence screening (Wu and Yin, 2015), conditional sure independence screening based on Cramer-von Mises statistic (Wang et al., 2017), and fused mean-variance filter (Yan et al., 2018). The aforementioned feature screening procedures mainly focus on the complete-data 
problems. However, in many fields such as medical, social and economic studies, there are some subjects with missing responses or predictors due to some reasons, such as, unwillingness of some sampled subjects to answer sensitivity questions, loss of information caused by uncontrollable factors, some scheduled visits intermittently or drop out of the study (Little and Rubin, 2002).

To address the issue mentioned above, there is an increasing literature on feature screening for ultrahigh dimensional data with missing data in recent years. For example, Lai et al. (2017) studied a model-free feature screening procedure for ultrahigh dimensional data with responses missing at random based on the inverse probability weighted (IPW) method, where the Kolmogorow filter method is adopted to screen the active predictors under an unknown propensity score function assumption. But, Lai et al.'s method heavily depends on the specification of unknown propensity score function, which is rather difficult to be correctly specified or efficiently estimated in the presence of ultrahigh dimensional predictors. To address the issue, Wang and Li (2018) proposed the missing indicator imputation screening approach and the Venn diagram-based screening procedure for ultrahigh dimensional data in the presence of missing responses at random. However, Wang and Li's method may not work well at some cases because missing values are imputed with the missing indicator value. Recently, Tang, Xia and Yan (2019) developed a feature screening method based on the profile marginal kernel-assisted 
estimating equations imputation technique in ultrahigh-dimensional partially linear models with missing responses at random, which is a model-based method. On the other hand, the MV-SIS method was developed for the case that response variable is the fully observed categorical data rather than missing indicator and predictors are continuous, and the DC-SIS method was proposed for the completely observed response and predictors and can not directly be utilized to screen features in the presence of missing responses and ultrahigh-dimensional predictors due to the "curse of dimensionality" issue in estimating the distribution function of missing response. To our knowledge, there is little work on applying the MV-SIS and DC-SIS methods to missing data problem for simultaneously screening important features associated with missing indicators and responses.

To solve the aforementioned problems, this paper proposes a novel two-step feature screening procedure by incorporating the idea of the MV-SIS and DC-SIS methods for ultrahigh-dimensional data with responses missing at random. The first step is to screen significant covariates associated with missing indicators by utilizing the fused mean-variance filter (Cui, Li and Zhong, 2015) to measure the dependence between missing indicators and covariates by regarding missing indicators as responses. Based on the selected significant covariates associated with missing indicator in the first step, the second step is to screen the important predictors associated with response by developing a modified distance correlation 
between marginal distributions of predictor and response variable with missing value, and nonparametrically imputing conditional distribution function of response. This step is heavily different from the DC-SIS procedure because it requires nonparametrically imputing missing responses, which may suffer from the well-known "curse of dimensionality" issue. To address this issue, a modified maximum ratio criterion for selecting the tuning parameter is proposed. The proposed tuning parameter selection procedure can choose a smaller model size than those given in Huang, Li and Wang (2014) and Ni and Fang (2016). The proposed feature screening procedure has the following merits. First, the proposed procedure is model free because it does not require specifying a regression model of response on predictors. Second, it can work well without resampling on the conditional distribution of missing value in that the kernel smoothing technique is applied to implement nonparametric conditional mean imputation. Third, it is robust to heavy-tailed distribution of response variable or outliers of response because only marginal distribution function of response is utilized to construct the utility for screening the significant predictors. Fourth, it is robust to the misspecification of propensity score function in that a special model for respondent probability is not required. Fifth, the modified tuning parameter selection procedure can effectively address the "curse of dimensionality" issue. Sixth, it possesses the sure screening properties under some regularity conditions. 
The rest of this paper is organized as follows. Section 2 introduces a new feature screening approach by fusing nonparametric conditional mean imputation and distance-correlation-based SIS procedure and mean-variance screening procedure, a modified tuning parameter selection procedure is also presented in Section 2. Section 3 investigates theoretical properties for the proposed feature screening approach under some regularity conditions. Simulation studies are conducted to investigate the finite sample performance of the proposed methodologies in Section 4. An example is used to illustrated the proposed methodologies in Section 5. A brief discussion is given in Section 6. Technical details are given in the Appendix.

\section{Feature screening approach}

\subsection{Distance correlation in the presence of missing responses at random}

Let $Y$ be response variable with a support $\Omega_{y}$, and $X=\left(X_{1}, \ldots, X_{p}\right)^{\top}$ be a p-dimensional predictor vector. Let $F(y \mid X)=\operatorname{Pr}(Y \leq y \mid X)$ be the conditional distribution function of $Y$ given $X$. Without specifying a regression model, we define the index sets of the active and inactive predictors as

$\mathcal{M}=\left\{k: F(y \mid X)\right.$ functionally depends on $X_{k}$ for some $\left.y \in \Omega_{y}\right\}$, $\mathcal{I}=\left\{k: F(y \mid X)\right.$ does not functionally depends on $X_{k}$ for any $\left.y \in \Omega_{y}\right\}$, respectively. The above definition indicates that $X_{k}$ is an active predictor if $k \in \mathcal{M}$, while $X_{k}$ is an inactive predictor if $k \in \mathcal{I}$. Denote $X_{\mathcal{M}}=\left\{X_{k}: k \in \mathcal{M}\right\}$ and $X_{\mathcal{I}}=\left\{X_{k}: k \in \mathcal{I}\right\}$ as the sets of the important and unimportant predictors, 
respectively. Here it is assumed that the dimensionality satisfies $p=o\left\{\exp \left(n^{\alpha}\right)\right\}$ for some constant $\alpha>0$, but the cardinality of $\mathcal{M}$, denoted as $|\mathcal{M}|$, satisfies $|\mathcal{M}|=o(n)$. In this framework, our main purpose is to screen important predictors $X_{\mathcal{M}}$ via some appropriate method.

Due to some merits of the distance-correlation-based sure independence screening (DC-SIS) procedure (Li, Zhong and Zhu, 2012), such as, it is equivalent to the marginal Pearson correlation learning for normal linear regression with normally distributed predictors, it is more effective than the marginal Pearson correlation learning in the presence of nonlinear relationship between two random vectors, it is model free and possesses the sure screening property, and its implementation does not involve any numerical optimization algorithm, we here consider screening features associated with response via the DC-SIS procedure. Following Li, Zhong and Zhu (2012) and Zhong et al. (2016), the marginal distance correlation between marginal distribution functions $F_{k}\left(X_{k}\right)$ of $X_{k}$ and $F(Y)$ of $Y$ can be defined as

$$
\omega_{k}=\operatorname{dcorr}^{2}\left\{F_{k}\left(X_{k}\right), F(Y)\right\}=\frac{\operatorname{dcov}^{2}\left\{F_{k}\left(X_{k}\right), F(Y)\right\}}{\operatorname{dcov}\left\{F_{k}\left(X_{k}\right), F_{k}\left(X_{k}\right)\right\} \operatorname{dcov}\{F(Y), F(Y)\}}
$$

for $k=1, \ldots, p$, where $F_{k}(x)=E\left\{I\left(X_{k} \leq x\right)\right\}$ for $k=1, \ldots, p$, and $F(y)=$ $E\{I(Y \leq y)\}$ in which $I(\cdot)$ denotes an indicator function, and $\operatorname{dcov}(u, v)$ represents the marginal distance covariance between two random variables $u$ and $v$ and is defined as $\operatorname{dcov}^{2}(u, v)=S_{1}+S_{2}-2 S_{3}$, where $S_{1}=E\{\|u-\tilde{u}\| \cdot\|v-\tilde{v}\|\}, S_{2}=$ $E\{\|u-\tilde{u}\|\} E\{\|v-\tilde{v}\|\}$ and $S_{3}=E\{E(\|u-\tilde{u}\| \mid u) E(\|v-\tilde{v}\| \mid v)\}$ in which $(\tilde{u}, \tilde{v})$ 
is an independent copy of $(u, v)$. Generally, $F_{k}\left(X_{k}\right)$ and $F(Y)$ are unknown and can be empirically estimated by $\hat{F}_{k}\left(X_{k}\right)=\frac{1}{n} \sum_{i=1}^{n} I\left(X_{i k} \leq X_{k}\right)$ and $\hat{F}_{n}(Y)=$ $n^{-1} \sum_{i=1}^{n} I\left(Y_{i} \leq Y\right)$ for the collected sample $\left\{\left(X_{i}, Y_{i}\right): i=1, \ldots, n\right\}$, respectively, where $X_{i}=\left(X_{i 1}, \ldots, X_{i p}\right)^{\top}$. In this case, $\omega_{k}$ can be estimated by the following sample distance correlation between $F_{k}\left(X_{k}\right)$ and $F(Y)$ :

$$
\hat{\omega}_{k}=\widehat{\operatorname{dcorr}}^{2}\left\{F_{k}\left(X_{k}\right), F(Y)\right\}=\frac{\widehat{\operatorname{dcov}}^{2}\left\{F_{k}\left(X_{k}\right), F(Y)\right\}}{\widehat{\operatorname{dcov}}\left(F_{k}\left(X_{k}\right), F_{k}\left(X_{k}\right)\right) \widehat{\operatorname{dcov}}\{F(Y), F(Y)\}}
$$

for $k=1, \ldots, p$, where $\widehat{\operatorname{dcov}}^{2}\left\{F_{k}\left(X_{k}\right), F(Y)\right\}=\hat{S}_{k 1}+\hat{S}_{k 2}-2 \hat{S}_{k 3}$ in which $\hat{S}_{k 1}=$ $n^{-2} \sum_{i=1}^{n} \sum_{j=1}^{n}\left|\hat{F}_{k}\left(X_{i k}\right)-\hat{F}_{k}\left(X_{j k}\right)\right| \cdot\left|\hat{F}_{n}\left(Y_{i}\right)-\hat{F}_{n}\left(Y_{j}\right)\right|, \hat{S}_{k 2}=n^{-4}\left\{\sum_{i=1}^{n} \sum_{j=1}^{n} \mid \hat{F}_{k}\left(X_{i k}\right)-\right.$ $\left.\hat{F}_{k}\left(X_{j k}\right) \mid\right\} \cdot\left\{\sum_{i=1}^{n} \sum_{j=1}^{n}\left|\hat{F}_{n}\left(Y_{i}\right)-\hat{F}_{n}\left(Y_{j}\right)\right|\right\}, \hat{S}_{k 3}=n^{-3} \sum_{i=1}^{n} \sum_{j=1}^{n} \sum_{l=1}^{n} \mid \hat{F}_{k}\left(X_{i k}\right)-$ $\hat{F}_{k}\left(X_{l k}\right)|\cdot| \hat{F}_{n}\left(Y_{j}\right)-\hat{F}_{n}\left(Y_{l}\right) \mid$. Li, Zhong and Zhu (2012) suggested selecting a set of important predictors with large $\hat{\omega}_{k}$ 's. Thus, the important predictors can be indexed as

$$
\hat{\mathcal{M}}=\left\{k: \hat{\omega}_{k} \geq c n^{-v} \quad \text { for } 1 \leq k \leq p\right\}
$$

for some pre-specified thresholds $c>0$ and $0 \leq v<1 / 2$.

When $Y_{i}^{\prime}$ 's are subject to missingness, the above presented DC-SIS procedure can not directly be used to select the important predictors. To this end, a new feature screening approach will be developed to simultaneously select the important predictors associated with $Y$ and important covariates associated with the missingness data mechanism as follows. 
Let $\delta_{i}=1$ if $Y_{i}$ is observed, and $\delta_{i}=0$ if $Y_{i}$ is missing. Throughout this paper, it is assumed that $\delta_{i}$ and $\delta_{j}$ are independent for any $i \neq j$, and $\delta_{i}$ only depends on $X_{i}$ such that $\operatorname{Pr}\left(\delta_{i}=1 \mid X_{i}, Y_{i}\right)=\operatorname{Pr}\left(\delta_{i}=1 \mid X_{i}\right)$ for $i=1, \ldots, n$, which indicates that the missingness data mechanism is MAR (Little and Rubin, 2002). In the ultrahigh dimensional setting, it is also assumed that only a small number of covariates in $X_{i}$ contribute to the missing indicator $\delta_{i}$. Again, without specifying a parametric model for $\operatorname{Pr}(\delta=1 \mid X)$, we define the index sets of the important and unimportant covariates associated with $\operatorname{Pr}(\delta=1 \mid X)$ as

$$
\begin{aligned}
& \mathcal{M}_{\delta}=\left\{k: \operatorname{Pr}(\delta=1 \mid X) \text { functionally depends on } X_{k}\right\} \\
& \mathcal{I}_{\delta}=\left\{k: \operatorname{Pr}(\delta=1 \mid X) \text { does not functionally depends on } X_{k}\right\}
\end{aligned}
$$

respectively, which shows that $X_{k}$ has an important effect on missing $Y$ when $k \in \mathcal{M}_{\delta}$, while $X_{k}$ does not associate with missing $Y$ when $k \in \mathcal{I}_{\delta}$. Similarly, $X_{\mathcal{M}_{\delta}}=\left\{X_{k}: k \in \mathcal{M}_{\delta}\right\}$ and $X_{\mathcal{I}_{\delta}}=\left\{X_{k}: k \in \mathcal{I}_{\delta}\right\}$ represent the sets of the important covariates associated with indicator $\delta$, and the unimportant covariates that does not associate with indicator $\delta$, respectively. Thus, the propensity score function can be written as

$$
\pi(Z)=\operatorname{Pr}(\delta=1 \mid X, Y)=\operatorname{Pr}(\delta=1 \mid X)=\operatorname{Pr}(\delta=1 \mid Z)
$$

where $Z=\left(Z_{1}, \ldots, Z_{s_{n}^{\delta}}\right)^{\top}, Z_{j}=X_{k}$ for some $k \in \mathcal{M}_{\delta}, s_{n}^{\delta}$ is the cardinality of $\mathcal{M}_{\delta}$.

Note that when response variable $Y$ is subject to MAR, the sample distance correlation defined in Equation 2.2 is not available because $\hat{F}_{n}(Y)$ can not be 
evaluated for missing $Y$. To address the issue, in what follows, a nonparametric mean imputation approach is developed to estimate $F(Y)$ under the assumption given in Equation (2.4).

Following Cheng and Chu (1996), if the missingness data mechanism model (2.4) is correctly specified, a nonparametric estimator of $F(y)$ is given as

$$
\hat{F}_{n}(y)=\frac{1}{n} \sum_{i=1}^{n}\left\{\delta_{i} I\left(Y_{i} \leq y\right)+\left(1-\delta_{i}\right) \hat{F}_{1 n}\left(y \mid Z_{i}\right)\right\}
$$

where $Z_{i}=\left(Z_{i 1}, \ldots, Z_{i s_{n}^{\delta}}\right)^{\top}$ in which $Z_{i j}=X_{i k}$ for some $k \in \mathcal{M}_{\delta}$, and $\hat{F}_{1 n}\left(y \mid Z_{i}\right)$ is a nonparametric regression estimator of $F_{1 n}\left(y \mid Z_{i}\right)=\operatorname{Pr}\left(Y \leq y \mid Z_{i}\right)$, which is defined as

$$
\hat{F}_{1 n}\left(y \mid Z_{i}\right)=\frac{\sum_{j=1}^{n} \delta_{j} I\left(Y_{j} \leq y\right) K\left(\frac{Z_{j 1}-Z_{i 1}}{h_{1}}, \ldots, \frac{Z_{j s_{n}^{\delta}}-Z_{i s_{n}^{\delta}}}{h_{s_{n}^{\delta}}}\right)}{\sum_{j=1}^{n} \delta_{j} K\left(\frac{Z_{j 1}-Z_{i 1}}{h_{1}}, \ldots, \frac{Z_{j s_{n}^{\delta}}-Z_{i s_{n}^{\delta}}}{h_{s_{n}^{\delta}}}\right)},
$$

where $K(\cdot)$ is a $s_{n}^{\delta}$-dimensionality kernel function, $h_{j}=C_{j} h$ is the bandwidth and $C_{j}$ is a fixed positive constant for $j=1, \ldots, s_{n}^{\delta}$, and $h=h_{n}^{0} \rightarrow 0$ as $n \rightarrow \infty$. It follows from Theorem 2.1 of Cheng and Chu (1996) that the estimator $\hat{F}_{n}(y)$ defined in Eq. 2.5 is a consistent estimator of $F(y)$. When $s_{n}^{\delta}$ is large, the preceding presented nonparametric estimator of $F_{1 n}\left(y \mid Z_{i}\right)$ may perform poorly. In this case, we can simply utilize a product of $s_{n}^{\delta}$ univariate kernel functions with independent smoothing parameters to replace $K(\cdot)$. In particular, when $s_{n}^{\delta}$ is diverging, we can adopt an adaptive kernel estimation to replace $\hat{F}_{1 n}\left(y \mid Z_{i}\right)$ (e.g., 
see Bouř, Kus and Franc, 2017). It is impossible to evaluate $\hat{F}_{n}(y)$ via Eq. 2.5 when the index set $\mathcal{M}_{\delta}$ involved is unknown. Hence, it is necessary to identify the index set $\mathcal{M}_{\delta}$ via some appropriate screening approach before evaluating $\hat{F}_{n}(y)$ via Eq. (2.5). Since $\delta$ is a bernoulli variable, identifying the index set $\mathcal{M}_{\delta}$ is equivalent to a binary discriminant analysis problem. In what follows, a fused mean-variance filter (Cui, Li and Zhong, 2015) is adopted to distinguish $\mathcal{M}_{\delta}$.

Denote $P_{0}=\operatorname{Pr}(\delta=0), P_{1}=\operatorname{Pr}(\delta=1)$ and $F_{k}(x)=\operatorname{Pr}\left(X_{k} \leq x\right)$. Let $F_{k 0}(x)=\operatorname{Pr}\left(X_{k} \leq x \mid \delta=0\right)$ and $F_{k 1}(x)=\operatorname{Pr}\left(X_{k} \leq x \mid \delta=1\right)$ be the conditional cumulative distribution function of $X_{k}$ given $\delta=0$ and $\delta=1$, respectively. Following Cui, Li and Zhong (2015), we define

$\omega_{k}^{\delta}=M V\left(X_{k} \mid \delta\right)=P_{0} \int\left\{F_{k 0}(x)-F_{k}(x)\right\}^{2} d F_{k}(x)+P_{1} \int\left\{F_{k 1}(x)-F_{k}(x)\right\}^{2} d F_{k}(x)$,

which is zero if $X_{k}$ is independent of $\delta$. The sample version of $\omega_{k}^{\delta}$ has the form of

$$
\hat{\omega}_{k}^{\delta}=\frac{1}{n} \sum_{j=1}^{n} \hat{P}_{0}\left\{\hat{F}_{k 0}\left(X_{j k}\right)-\hat{F}_{k}\left(X_{j k}\right)\right\}^{2}+\frac{1}{n} \sum_{j=1}^{n} \hat{P}_{1}\left\{\hat{F}_{k 1}\left(X_{j k}\right)-\hat{F}_{k}\left(X_{j k}\right)\right\}^{2}
$$

where $\hat{P}_{0}=n^{-1} \sum_{i=1}^{n} I\left(\delta_{i}=0\right), \hat{P}_{1}=n^{-1} \sum_{i=1}^{n} I\left(\delta_{i}=1\right), \hat{F}_{k}(x)=n^{-1} \sum_{i=1}^{n} I\left(X_{i k} \leq\right.$ $x), \hat{F}_{k 0}(x)=n^{-1} \sum_{i=1}^{n} I\left(X_{i k} \leq x, \delta_{i}=0\right) / \hat{P}_{0}$, and $\hat{F}_{k 1}(x)=n^{-1} \sum_{i=1}^{n} I\left(X_{i k} \leq\right.$ $\left.x, \delta_{i}=1\right) / \hat{P}_{1}$. Thus, $\hat{\omega}_{k}^{\delta}$ can be used to select the important covariates indexed by

$$
\hat{\mathcal{M}}_{\delta}=\left\{k: \hat{\omega}_{k}^{\delta} \geq c_{\delta} n^{-\tau_{\delta}} \text { for } 1 \leq k \leq p\right\}
$$

where $c_{\delta}$ and $\tau_{\delta}$ are the pre-determined thresholds defined in Condition (C2) below. However, in many practical applications, it is quite difficult to give the thresholds 
$c_{\delta}$ and $\tau_{\delta}$. To this end, we consider the following reduced subset

$$
\hat{\mathcal{M}}_{\delta}^{*}=\left\{k: \hat{\omega}_{k}^{\delta} \text { is among the top } d_{\delta} \text { largest of all }\right\}
$$

where $d_{\delta}<n$ is a pre-specified positive integer. The above presented feature screening procedure is called the MV-based sure independence screening (denoted as 'MV-SIS'). By the MV-SIS procedure, the number of ultrahigh dimensional covariates is reduced to $d_{\delta}$. Under conditions $(\mathrm{C} 1)$ and $(\mathrm{C} 2)$ given below, it follows from Theorem 2.1 of Cui, Li, and Zhong (2015) that $\operatorname{Pr}\left(\mathcal{M}_{\delta} \subseteq \hat{\mathcal{M}}_{\delta}\right) \rightarrow 1$, which shows that utilizing the above presented MV-SIS procedure to select the important covariates associated with $\delta$ has our desirable sure screening property.

Based on the selected subset $\hat{\mathcal{M}}_{\delta}^{*}$, Eq. 2.5 can be rewritten as

$$
\hat{F}_{n}(y)=\frac{1}{n} \sum_{i=1}^{n}\left\{\delta_{i} I\left(Y_{i} \leq y\right)+\left(1-\delta_{i}\right) \hat{F}_{1 n}\left(y \mid \hat{Z}_{i}\right)\right\},
$$

where $\hat{Z}_{i}=\left(Z_{i 1}, \ldots, Z_{i \hat{s}_{n}^{\delta}}\right)^{\top}, Z_{i j}=X_{i k}$ for some $k \in \hat{\mathcal{M}}_{\delta}^{*}$, and $\hat{s}_{n}^{\delta}$ is the cardinality of $\hat{\mathcal{M}}_{\delta}^{*}$.

With the estimated $\hat{F}_{n}(y)$ together with $\hat{\omega}_{k}$ defined in Eq. 2.2, we can obtain the selected subset $\hat{\mathcal{M}}$ via Eq. 2.3. Similarly, it is quite difficult to specify thresholds $c$ and $v$. Again, we utilize the following criterion to select the significant predictors:

$$
\hat{\mathcal{M}}^{*}=\left\{k: \hat{\omega}_{k} \text { is among the top } d_{n} \text { largest of all }\right\} \text {, }
$$


where $d_{n}<n$ is a pre-specified threshold. The preceding developed feature screening procedure is referred to as the nonparametric-mean-imputation based DC-SIS procedure (denoted as 'DCNI-SIS').

Remark 1. When the sample values of the mean-variance utility corresponding to important covariates (i.e., large $\hat{\omega}_{k}^{\delta}$ 's) are always ranked beyond those corresponding to unimportant covariates with a relatively high probability, the following modified tuning parameter selection algorithm can select a relatively small model size to include all important covariates. In this case, it is not necessary to employ the penalized likelihood methods to further reduce the number of covariates. Thus, the modified tuning parameter selection algorithm can effectively address the issue of "curse of dimensionality". However, when $\hat{\omega}_{k}^{\delta}$ 's corresponding to important covariates are not always ranked beyond those for inactive covariates, the number of the subset $\widehat{\mathcal{M}}_{\delta}^{*}$ may be still quite large, which implies that there is still the "curse of dimensionality" problem. To this end, the penalized likelihood approaches (e.g., the SCAD and adaptive Lasso methods) may be adopted to further select important covariates. Thus, the estimated subset $\widehat{\mathcal{M}}_{\delta}^{*}$ may further be reduced to the subset $\widetilde{\mathcal{M}}_{\delta}^{*}$ satisfying $\operatorname{Pr}\left(\mathcal{M}_{\delta} \subseteq \widetilde{\mathcal{M}}_{\delta}^{*}\right) \rightarrow 1$ as $n \rightarrow \infty$.

\subsection{The selection of tuning parameter $d_{\delta}$}

In many practical applications, we require determining an optimal tuning parameter $d_{\delta}$ in Equation (2.6). For simplicity, $d_{\delta}$ is abbreviated as $d$, and $d_{0}$ repre- 
sents the true size of the considered model. Fan and Lv (2008) suggested taking $d=\lfloor n / \log n\rfloor$, where the notation $\lfloor a\rfloor$ denotes the integer that is less than or equal to $a$. In this case, for our considered nonparametric mean imputation, there is still the "curse of dimensionality" problem. To address the issue, Huang, Li and Wang (2014) proposed an approach to select the tuning parameter based on the maximum ratio criterion, but their method may lead to a much larger $d$ than $d_{0}$. To solve this problem, Ni and Fang (2016) proposed a modified approach of Huang, Li and Wang (2014) to select the tuning parameter, which may be unstable.

To address the aforementioned issue, we here develop a modified approach of $\mathrm{Ni}$ and Fang (2016). Let $\left\{t_{1}, \ldots, t_{p}\right\}$ be a permutation of the set $\{1, \ldots, p\}$ such that $\hat{\omega}_{t_{1}} \geq \ldots \geq \hat{\omega}_{t_{p}}$, where $\hat{\omega}_{t_{k}}$ may represent $\hat{\omega}_{k}$ or $\hat{\omega}_{k}^{\delta}$ for $k=1, \ldots, p$. Generally, an optimal value of $d$ should be selected on the basis of the following two conditions: (i) $\left(\hat{\omega}_{t_{k}}+\hat{\omega}_{t_{k+1}}\right) /\left(\hat{\omega}_{t_{k+1}}+\hat{\omega}_{t_{k+2}}\right)$ should be $O_{p}(1)$ for $k \neq d_{0}$, and (ii) $\left(\hat{\omega}_{t_{k}}+\hat{\omega}_{t_{k+1}}\right) /\left(\hat{\omega}_{t_{k+1}}+\hat{\omega}_{t_{k+2}}\right) \stackrel{P}{\rightarrow} \infty$ for $k=d_{0}$, where $\stackrel{P}{\rightarrow}$ represents the convergence in probability. Thus, similar to Ni and Fang (2016), we take

$$
d=\arg \max _{1 \leq k \leq p-2} \frac{\hat{\omega}_{t_{k}}+\hat{\omega}_{t_{k+1}}}{\hat{\omega}_{t_{k+1}}+\hat{\omega}_{t_{k+2}}} .
$$

In this case, $d$ can be selected such that $d_{\min } \leq d \leq d_{\max }$, where $d_{\min } \geq 1$ and $d_{\max }<p$ are two user-specified positive integers. The algorithm for implementing the aforementioned approach is as follows.

Step 1. Calculate $d^{(1)}=\arg \max _{1 \leq k \leq d_{\max }}\left\{\left(\hat{\omega}_{t_{k}}+\hat{\omega}_{t_{k+1}}\right) /\left(\hat{\omega}_{t_{k+1}}+\hat{\omega}_{t_{k+2}}\right)\right\}$. 
Step 2: For $m=1,2, \ldots$, if $d^{(m)}<d_{\min }$, thus calculate

$d^{(m+1)}=\arg \max _{d^{(m)}+1 \leq k \leq d_{\max }}\left\{\left(\hat{\omega}_{t_{k}}+\hat{\omega}_{t_{k+1}}\right) /\left(\hat{\omega}_{t_{k+1}}+\hat{\omega}_{t_{k+2}}\right)\right\}$.

Step 3: Repeat step 2 until $d^{(m)} \geq d_{\text {min }}$ and choose $d=d^{(m)}$.

Intuitively, the above presented procedure is expected to work well because the modified algorithm is more robust than that given in $\mathrm{Ni}$ and Fang (2016). In practical applications, similar to $\mathrm{Ni}$ and Fang (2016), we may take $d_{\max }$ as $n$ or $O(n / \log n)$, which is a commonly used value in the feature screening literature. To be conservative, we may take $d_{\min }=2,5$ or even larger. However, the above presented procedure may lead to select some other inactive predictors. To solve this problem, one can again utilize the regularization method to select active predictors.

\section{Theoretical properties}

Now we discuss theoretical properties of the above proposed DCNI-SIS procedure. To this end, we first introduce some conditions used below.

(C1) There are two positive constants $c_{1}$ and $c_{2}$ such that $c_{1} \leq \min \left\{P_{0}, P_{1}\right\} \leq$ $\max \left\{P_{0}, P_{1}\right\} \leq c_{2}$, where $P_{t}=\operatorname{Pr}(\delta=t)$ for $t=0,1$.

(C2) There are two positive constants $c_{\delta}>0$ and $0 \leq \tau_{\delta}<1 / 2$ such that $\min _{k \in \mathcal{M}_{\delta}} \omega_{k}^{\delta} \geq 2 c_{\delta} n^{-\tau_{\delta}}$

(C3) The probability density function $f(Z)$ is bounded away from $\infty$ on the support $\mathbb{Z}$ of $Z$, and has bounded partial derivatives (within the compact support of $Z$ ) up to two order. 
(C4) The propensity score function $\pi(Z)=\operatorname{Pr}(\delta=1 \mid Z)$ satisfies $\min _{i} \pi\left(Z_{i}\right) \geq$ $D_{0}>0$ a.s. for some positive constant $D_{0}$, and has bounded partial derivatives (within the compact support of $Z$ ) up to order 2 .

(C5) Function $K(\cdot)$ is a $s_{n}^{\delta}$-dimensionality kernel function of order $\kappa$ satisfying $\int K\left(u_{1}, \ldots, u_{s_{n}^{\delta}}\right) d u_{1} \cdots d u_{s_{n}^{\delta}}=1, \int u_{j}^{l} K\left(u_{1}, \ldots, u_{s_{n}^{\delta}}\right) d u_{1} \cdots d u_{s_{n}^{\delta}}=0$ for any $1 \leq$ $l \leq \kappa$, and $\int u_{j}^{\kappa} K\left(u_{1}, \ldots, u_{s_{n}^{\delta}}\right) d u_{1} \cdots d u_{s_{n}^{\delta}} \neq 0$ for any $j=1, \ldots, s_{n}^{\delta}$, where $s_{n}^{\delta}$ is the dimension of $Z$. In particular, consider $K(\cdot)=\prod_{i=1}^{s_{n}^{\delta}} K_{i}(\cdot)$. For $\forall i \in\left\{1, \ldots, s_{n}^{\delta}\right\}$, kernel function $K_{i}(\cdot)$ is the probability density function such that (i) it is bounded and has compact support; (ii) it is symmetric with $\sigma^{2}=\int \omega^{2} K_{i}(\omega) d \omega<\infty$; (iii) $K_{i}(\omega) \geq D_{1}$ for some constant $D_{1}>0$ in some closed interval centered at zero.

(C6) For $j=1, \ldots, s_{n}^{\delta}, h_{j}=C_{j} h$ is the bandwidth in which $C_{j}$ is some fixed positive constant, and $h=O\left\{n^{-\theta /\left(s_{n}^{\delta}+2\right)}\right\}$ for some $0<\theta \leq 1$.

(C7) There are two positive constants $c>0$ and $0<v<1 / 3$ such that $\min _{k \in \mathcal{M}} \omega_{k} \geq 2 c n^{-v}$

Condition ( $\mathrm{C} 1$ ) is used to ensure that the proportions of $\delta=0$ and $\delta=1$ are neither too small nor too large, and has been utilized in Shao, Yu and Zhou (2016). Condition (C2) requires that the minimum true signal cannot be too small and its order is $n^{-\tau_{\delta}}$, which allows the minimum true signal to degenerate to zero as $n \rightarrow \infty$, and has been used in Cui, Li and Zhong (2015). Conditions (C1) and (C2) ensure that all the important covariates associated with missing indicator $\delta$ can be 
selected by the aforementioned DCNI-SIS procedure with probability tending to 1. Conditions (C3)-(C6) are widely adopted in nonparametric and missing data literature (Tang et al., 2014). Condition (C7) is employed to ensure that the values of the marginal DCNI-SIS utility corresponding to active predictors can not be too small. Condition (C7) has been used in the ultrahigh dimensional data analysis literature (Li, Zhong and Zhu, 2012).

Based on the aforementioned conditions, we obtain the following theorem.

Theorem 1. Suppose that Conditions (C1)-(C7) and $\mathcal{M}_{\delta} \subseteq \widehat{\mathcal{M}}_{\delta}$ hold. For any $v / 2<\alpha<1 / 2-v$ and $0 \leq v-\alpha<\theta \leq 1$, and $c_{2} \leq c_{3} n^{-\alpha}$ with $c_{3}>0$, there exists positive constants $m_{1}, m_{2}$ and $m_{3}$ such that

$$
\operatorname{Pr}\left(\max _{1 \leq k \leq p}\left|\hat{\omega}_{k}-\omega_{k}\right| \geq c n^{-v}\right) \leq O\left(p \mathbb{J}_{n}\right)
$$

where $\mathbb{J}_{n}=\exp \left\{-m_{1} n^{1-2(v+\alpha)}\right\}+n^{2-\alpha} \exp \left\{-m_{2} n^{1-2(v-\alpha)}\right\}+n \exp \left(-m_{3} n^{\alpha}\right)$. In particular, we have

$$
\operatorname{Pr}(\mathcal{M} \subseteq \widehat{\mathcal{M}}) \geq 1-O\left(s_{n} \mathbb{J}_{n}\right)
$$

which indicates that $\operatorname{Pr}(\mathcal{M} \subseteq \widehat{\mathcal{M}}) \rightarrow 1$ as $n \rightarrow \infty$, where $s_{n}$ is the cardinality of $\mathcal{M}$

Theorem 1 shows that the above proposed DCNI-SIS procedure has the desirable sure screening property. Thus, we extend the DC-SIS procedure to ultrahigh dimensional data with responses missing at random. 


\section{Simulation studies}

In this section, simulation studies are conducted to investigate the finite sample performance of the above proposed feature screening procedure. The following criteria are used to evaluate the performance of feature screening procedure: AMS (i.e., the average number of model sizes); CF (i.e., the proportion that all the true active predictors are exactly selected among 500 replications); OF (i.e., the proportion that all the true active covariates are exactly selected and at least one inactive covariate is selected among 500 replications); UF (i.e., the proportion that the true active covariates are not completely selected among 500 replications); $\mathrm{CP}_{j}$ (i.e., the proportion that the true active predictor $X_{j}$ is selected into the submodel with size $\lfloor n / \log (n)\rfloor$ among 500 replications); $\mathrm{CP}_{a}$ (i.e., the proportion that all the true active predictors are selected into the submodel with size $\lfloor n / \log (n)\rfloor$ among 500 replications); MMS (i.e., the minimum model size needed to include all the true active predictors). Generally, some SIS procedure is regarded as better than other SIS procedures if the MMS value for the former is closer to the true number of model size than those for the latter, the $\mathrm{CF}, \mathrm{CP}_{j}$ and $\mathrm{CP}_{a}$ values for the former is larger than those for the latter, the AMS, OF and UF values for the former are smaller than those for the latter. We consider the following three experiments with $(p, n)=(1000,200)$, and set the screening size as $d_{n}=\lfloor n / \log (n)\rfloor$.

For comparison, we evaluate the results for the SIS (Fan and Lv, 2008), SIRS 
(Zhu et al., 2011), DC-SIS (Li, Zhong and Zhu, 2012), DF-SIS (Wu and Yin, 2015), MC-SIS (Wang et al., 2017), BMI procedures (e.g., MI-I, MI-S and V-D methods) applying DC-SIS method to select predictors associated with $Y$ (Wang and Li, 2018), and the complete-case (the 'CC') analysis method with the fixed screening size $d_{n}$ under several different missingness data mechanism models.

Experiment 1 This experiment is designed to investigate the finite sample performance of the proposed DCNI-SIS under the assumption that $E(Y \mid X)$ is linear in some components of $X=\left(X_{1}, \ldots, X_{p}\right)^{\top}$. Here we generate response $Y$ from the following linear regression model: $Y=X^{\top} \beta+\varepsilon$, where $\beta=\left(\beta_{1}, \ldots, \beta_{p}\right)^{\top}=$ $(2.5,2.0,4.0,2.0,0.0, \ldots, 0.0)^{\top}$, which indicates that $X_{1}, \ldots, X_{4}$ are active predictors and $X_{5}, \ldots, X_{p}$ are inactive predictors, and $X=\left(X_{1}, \ldots, X_{p}\right)^{\top}$ is generated from multivariate normal distribution with mean zero and covariance matrix $\Sigma=\left(\sigma_{i j}\right)$ in which $\sigma_{i j}=0.5^{|i-j|}$. To demonstrate that the proposed DCNI-SIS procedure does not depend on the distribution of predictors, $X_{1}$ and $X_{4}$ are replaced by those generated from the normal distribution $N(0,2)$; and it is robust to outliers of response, we add $5 \%$ outliers which generated from the distribution $U(70,90)$ for response. Also, to illustrate that the proposed DCNI-SIS procedure is independent of the distribution of $\varepsilon$, we consider the following three settings: (E1) $\varepsilon \sim N(0,1),(\mathrm{E} 2) \varepsilon \sim t(3)$, where $t(3)$ represents the $t$-distribution with three degrees of freedom, and (E3) $\varepsilon \sim$ Cauchy $(0,1)$, where Cauchy $(0,1)$ denotes 
the Cauchy distribution with location and scatter parameters being 0 and 1 . It is assumed that $X$ is completely observed, while $Y$ is subject to missingness.

To create missing data for $Y$, missing indicator $\delta$ is generated from Bernoulli distribution with probability $\pi=\operatorname{Pr}(\delta=1 \mid Z)$ specified by

(i) $\operatorname{logit}(\pi)=\alpha_{0}+\alpha_{1} X_{3}$, where $\left(\alpha_{0}, \alpha_{1}\right)=(-1.0,4.0), \operatorname{logit}(a)=\log \{a /(1-a)\}$;

(ii) $\pi=0.15$ if $X_{2}+X_{3}<0$, and 0.85 otherwise;

(iii) $\operatorname{logit}(\pi)=\alpha_{0}+\alpha_{1} X_{3}+\alpha_{2} Y$, where $\left(\alpha_{0}, \alpha_{1}, \alpha_{2}\right)=(-1.1,4.0,0.01)$.

Scenario (i) describes a MAR mechanism specified by a logistic regression model, and indicates $Z=X_{3}$, i.e., the number of the important covariates associated with missing indicator $\delta$ is 1 . Scenario (ii) represents a MAR mechanism nonparametrically specified, and implies $Z=\left(Z_{1}, Z_{2}\right)^{\top}=\left(X_{2}, X_{3}\right)^{\top}$, i.e., the number of the important covariates associated with missing indicator $\delta$ is 2 . Scenario (iii) denotes a nonignorable missing data mechanism and prescribes a selection bias case, which is used to investigate the robustness of the proposed DCNI-SIS procedure to the misspecification of $\pi$, and implies $Z=X_{3}$, i.e., the number of the important covariates associated with missing indicator $\delta$ is 1 . The average missing proportions among 500 replications corresponding to the above presented three missingness data mechanisms (i), (ii) and (iii) are about $(56.3 \%, 55.8 \%, 56.2 \%),(50.4 \%, 49.9 \%$, $49.9 \%$ ) and $(56.6 \%, 56.2 \%, 56.7 \%)$ for error distributions (E1), (E2) and (E3), respectively. 
For each of 500 datasets generated from each of the above considered nine settings (i.e., three error distributions $\times$ three missingness data mechanisms), the preceding proposed MV-SIS and DCNI-SIS procedures are used to screen important covariates associated with $\delta$ and active predictors in the considered linear regression model, respectively. To estimate the distribution function of $Y$ in the presence of missing $Y$, we take the kernel function $K(\cdot)=\prod_{j=1}^{s_{n}^{\delta}} K_{j}(\cdot)$ with $K_{j}\left(z_{j}\right)=0.75(1-$ $\left.z_{j}^{2}\right)_{+}$, and set the bandwidth as $h_{j}=C_{j} \hat{\sigma}_{z_{j}} n^{-1 / 5}$, where $C_{j}=1$, and $\hat{\sigma}_{z_{j}}$ is the standard deviation of observations $\left\{Z_{i j}: i=1, \ldots, n ; j=1, \ldots, s_{n}^{\delta}\right\}$.

Results for screening the important covariates in propensity score function are given in Table 1, where 'MV-TM' and 'MV-T' represent the MV-SIS approaches with $d_{\min }=1$ for scenarios (i) and (iii) or $d_{\min }=2$ for scenario (ii), and $d_{\delta}$ determined by the modified tuning parameter selection method introduced in Section 2.2 and the algorithm introduced in Ni and Fang (2016), respectively. Examination of Table 1 shows that the MV-TM method behaves better than the MV-T method because the former has larger $\mathrm{CF}$ values, and smaller OF values than the latter, and the AMS values for the former are closer to the true model size than those for the latter regardless of error distributions and missingness data mechanisms.

Results for identifying active predictors are presented in Table 2, where 'DCNITM' and 'DCNI-T' represent the DCNI-SIS procedures with $d_{\min }=4$ and $d_{n}$ determined by the modified tuning parameter selection method introduced in Section 
2.2 and the algorithm introduced in $\mathrm{Ni}$ and Fang (2016), respectively. Examination of Table 2 evidences that the DCNI-TM method behaves better than the DCNI-T method in that the former has larger CF values, and smaller OF values than the latter, and the AMS values for the former are closer to the true model size than those for the latter regardless of error distributions and missingness data mechanisms.

Results for $C P_{1}, \ldots, C P_{4}$ and $C P_{a}$ in identifying active predictors with the fixed $d_{n}=\lfloor n / \log (n)\rfloor$ are reported in Table 3. Inspection of Table 3 indicates that the DCNI-SIS procedure outperforms other feature screening procedures in that the former has largest $\mathrm{CP}_{a}$ value among ten compared feature screening methods regardless of error distributions and missingness data mechanisms.

Results for $5 \%, 25 \%, 50 \%, 75 \%$ and $95 \%$ quantiles of minimum model sizes for various feature screening methods are presented in Table 4, which indicates that the DNCI-SIS procedure outperforms other feature screening procedures in identifying active predictors because all the quantiles of minimum model sizes for the former are closest to the true model size among ten compared feature screening methods. That is, the DCNI-SIS procedure is more efficient in selecting active predictors than other feature screening procedures.

Experiment 2 This experiment is designed to investigate the performance of the proposed DCNI-SIS under the assumption that $E(Y \mid X)$ is nonlinear in some 
components of $X$. In this experiment, we generate response $Y$ from the following regression model:

$$
Y=\beta_{1} I\left(X_{1}>0\right)+\beta_{2} X_{2} X_{4}+\beta_{3}\left|\sin \left(X_{3}\right)\right|+\beta_{4} X_{4}^{2}+\varepsilon
$$

where $\beta=\left(\beta_{1}, \ldots, \beta_{p}\right)^{\top}=(4,4.5,3.5,2.5,0.0, \ldots, 0.0)^{\top}$, which implies that $X_{1}, \ldots$, $X_{4}$ are active predictors and $X_{5}, \ldots, X_{p}$ are inactive predictors, and $X=\left(X_{1}, \ldots, X_{p}\right)^{\top}$ is generated from multivariate normal distribution with zero mean and covariance matrix $\Sigma=\left(\sigma_{i j}\right)$ with $\sigma_{i j}=0.5^{|i-j|}$, and $\varepsilon$ is independently generated as in Experiment 1. It is assumed that $X$ is completely observed, while $Y$ is subject to missingness.

Similar to Experiment 1, missing indicator $\delta$ for $Y$ is generated from Bernoulli distribution with probability $\pi=\operatorname{Pr}(\delta=1 \mid Z)$ specified by

(i) $\pi=0.2$ if $\left|X_{3}-1\right| \leq 1$, and 0.8 otherwise;

(ii) $\operatorname{logit}(\pi)=\alpha_{0}+\alpha_{1} X_{2}+\alpha_{2} X_{3}$ with $\left(\alpha_{0}, \alpha_{1}, \alpha_{2}\right)=(-1.0,-4.0,-5.0)$;

(iii) $\pi=0.2$ if $\left|X_{3}-0.02 Y-0.9\right| \leq 1.5$, and 0.8 otherwise.

Scenarios (i) and (ii) represent MAR mechanisms for missing response $Y$. Scenario (iii) is a nonignorable missing mechanism and is designed to investigate the robustness of the DCNI-SIS procedure to misspecification of missingness data mechanism. Thus, the numbers of the important covariates associated with $\delta$ for Scenarios (i), (ii) and (iii) are 1, 2 and 1, respectively. The average missing proportions for Scenarios (i), (ii) and (iii) are about $(48.5 \%, 48.5 \%, 48.5 \%),(51.1 \%, 51.1 \%, 50.8 \%)$ 
and $(60.8 \%, 60.8 \%, 60.4 \%)$ for error distributions (E1), (E2) and (E3), respectively.

For each of 500 datasets generated from each of the above considered three error distributions together with three missingness data mechanisms, the preceding proposed DCNI-SIS procedure together with other methods are used to distinguish active predictors in the considered nonlinear regression model and propensity score function. To estimate $F(Y)$ for missing $Y$, we take the same kernel function $K(u)$ as in Experiment 1. The same value of $d_{\min }$ is taken as that given in Experiment 1. To save space, results for identifying active covariates associated with missing indicator and important predictors associated with response are reported in Tables S1-S4 of Supplementary Materials. Examination of Tables S1-S4 yields the similar observations as those given in Experiment 1.

Experiment 3 This experiment is designed to investigate the finite sample performance of the proposed DCNI-SIS under the assumption that $E(Y \mid X)$ has interaction terms on some components of $X$, and $\mathcal{M}_{\delta} \cap \mathcal{M}$ is empty or $\mathcal{M}_{\delta} \supset \mathcal{M}$. In this experiment, we generate the response from the following regression model:

$$
Y=\exp \left(\beta_{1} X_{1}^{2}\right)+\exp \left(\beta_{2} X_{1} X_{2}\right)+\exp \left(\beta_{3} X_{2} X_{3}\right)+\varepsilon
$$

where $\beta=\left(\beta_{1}, \ldots, \beta_{p}\right)^{\top}=(3.0,3.0,3.0,0.0, \ldots, 0.0)^{\top}$, and $X=\left(X_{1}, \ldots, X_{p}\right)^{\top}$ and $\varepsilon$ are independently generated as in Experiment 2. It is assumed that $X$ is completely observed, while $Y$ is subject to missingness.

Similar to Experiment 1, missing indicator $\delta$ for $Y$ is generated from a Bernoulli 
distribution with probability $\pi=\operatorname{Pr}(\delta=1 \mid Z)$ specified by

(i) $\operatorname{logit}(\pi)=\alpha_{0}+\alpha_{1} X_{3}+\alpha_{2} X_{600}$, where $\left(\alpha_{0}, \alpha_{1}, \alpha_{2}\right)=(-1.0,-4.0,-4.0)$;

(ii) $\operatorname{logit}(\pi)=\alpha_{0}+\alpha_{1} X_{4}+\alpha_{2} X_{5}$, where $\left(\alpha_{0}, \alpha_{1}, \alpha_{2}\right)=(-1.0,-3.0,-3.0)$;

(iii) $\operatorname{logit}(\pi)=\alpha_{0}+\alpha_{1} X_{1}+\alpha_{2} X_{2}+\alpha_{3} X_{3}+\alpha_{4} X_{4}$, where $\left(\alpha_{0}, \alpha_{1}, \alpha_{2}, \alpha_{3}, \alpha_{4}\right)=$ $(-1.0,-4.0,-4.0,-4.0,-4.0)$.

Scenarios (i) -(iii) describe a MAR mechanism for missing response $Y$. Scenario (i) shows that $\mathcal{M}_{\delta} \cap \mathcal{M}$ is not empty, Scenario (ii) indicates that $\mathcal{M}_{\delta} \cap \mathcal{M}$ is empty, and Scenario (iii) implies $\mathcal{M}_{\delta} \supset \mathcal{M}$. The average missing proportions corresponding to the above presented three missingness data mechanisms (i), (ii) and (iii) are about $(53.3 \%, 52.9 \%, 53.2 \%),(53.9 \%, 53.9 \%, 53.9 \%)$ and $(49.4 \%, 49.2 \%, 48.9 \%)$ for error distributions (E1), (E2) and (E3), respectively.

Again, for each of 500 datasets generated from each of the above considered three distributions for random error together with three missingness data mechanisms, the preceding proposed DCNI-SIS procedure together with other methods are used to screen active predictors in the considered nonlinear model and propensity score function. In estimating $F(Y)$ for missing $Y$, we take the same kernel function $K(u)$ as in Experiment 1, set the bandwidth as $h_{j}=C_{j} \hat{\sigma}_{z_{j}} n^{-1 / 5}$ and $C_{j}=1$ for Scenarios (i) and (ii), and set $h_{j}=\hat{\sigma}_{z_{j}} n^{-1 / 6}$ for Scenario (iii). For MVSIS method, we take $d_{\text {min }}=2$ for scenarios (i) and (ii), and $d_{\text {min }}=4$ for scenario (iii). For DCNI-SIS method, we take $d_{\min }=3$. To save space, results are given in 
Tables S5-S8 of Supplementary Materials. Inspection of Tables S5-S8 shows the similar observations as in Experiment 1, which implies that the proposed DCNISIS procedure can be used to identify linear or nonlinear relationships between response and predictors.

Experiment 4 This experiment is designed to investigate the finite sample performance of the proposed DCNI-SIS method in the presence of discrete responses. To this end, we generate the response $Y$ from a binomial distribution $\operatorname{Binomial}(5, \theta)$ with the probability $\theta=\exp (a) /(1+\exp (a))$, where $a=\beta_{1} X_{1}+$ $\sin \left(\beta_{2} X_{2}+\beta_{3} X_{3}+\beta_{4} X_{4}\right)$, and $\beta=\left(\beta_{1}, \ldots, \beta_{p}\right)^{\top}=(2.0,3.0,2.5,2.8,0.0, \ldots, 0.0)^{\top}$ which indicates that $X_{1}, \ldots, X_{4}$ are active predictors and $X_{5}, \ldots, X_{p}$ are inactive predictors. Here $X=\left(X_{1}, \ldots, X_{p}\right)^{\top}$ is generated from multivariate normal distribution with mean zero and covariance matrix $\Sigma=\left(\sigma_{i j}\right)$ with $\sigma_{i j}=0.5^{|i-j|}$. It is assumed that $X$ is completely observed, while $Y$ is subject to missingness.

To create missing data for $Y$, missing indicator $\delta$ is generated from Bernoulli distribution with probability $\pi=\operatorname{Pr}(\delta=1 \mid Z)$ specified by

(i) $\operatorname{logit}(\pi)=\alpha_{0}+\alpha_{1} X_{3}+\alpha_{1} X_{4}$, where $\left(\alpha_{0}, \alpha_{1}, \alpha_{2}\right)=(-1.0,-4.0,-4.0)$;

(ii) $\operatorname{logit}(\pi)=\alpha_{0}+\alpha_{1} X_{4}+\alpha_{2} X_{5}$, where $\left(\alpha_{0}, \alpha_{1}, \alpha_{2}\right)=(-1.0,-4.0,-4.0)$.

Scenarios (i) and (ii) describe a MAR mechanism for missing response $Y$. Scenario (i) shows $\mathcal{M}_{\delta} \subset \mathcal{M}$, Scenario (ii) indicates that $\mathcal{M}_{\delta} \cap \mathcal{M}$ is not empty. The average missing proportions among 500 replications corresponding to the above presented 
two missingness data mechanisms (i) and (ii) are about $52.0 \%$ and $51.9 \%$, respectively. To estimate $F(Y)$ for missing $Y$, we take the same kernel function $K(u)$ as in Experiment 1. The same value of $d_{\min }$ is taken as that given in Experiment 3 regardless of MV-SIS and DCNI-SIS methods. To save space, results are given in Tables S9-S12 of Supplementary Materials. Inspection of Tables S9-S12 shows that the proposed DCNI-SIS procedure can identify relationships between response (or indicators) and predictors well in the presence of discrete responses.

\section{An example}

In this section, the cardiomyopathy microarray dataset, which has been analyzed by Li, Zhong and Zhu (2012), Zhong et al. (2016) and Wang and Li (2018), is used to illustrate the preceding proposed DCNI-SIS approach. The main purpose of this study is to identify the most influential genes for overexpression of a $\mathrm{G}$ protein-coupled receptor (Ro1). As an illustration, we take the Ro1 expression level as response variable $Y$, while other 6319 gene expression levels are taken as predictors, i.e., $X=\left(X_{1}, \ldots, X_{6319}\right)^{\top}$. There is only 30 specimens observed (i.e., $n=30$ ), which indicates that $n$ is rather small and $p=6319$ is sufficiently large. Li, Zhong and Zhu (2012) identified the most important two genes (e.g., Msa.2134.0 and Msa.2877.0) via the DC-SIS procedure. In this dataset, $Y$ and $X$ are completely observed. To demonstrate the application of the above proposed 
DCNI-SIS procedure, we artificially assume that $Y$ is MAR, and missing values for $Y$ are generated by the following propensity score function:

$$
\operatorname{Pr}(\delta=1 \mid Y, X)=\operatorname{Pr}(\delta=1 \mid Z)=\frac{\exp \left(\alpha_{0}+\alpha_{1} X_{1}+\alpha_{2} X_{268}\right)}{1+\exp \left(\alpha_{0}+\alpha_{1} X_{1}+\alpha_{2} X_{268}\right)},
$$

where $Z=\left(X_{1}, X_{268}\right)^{\top}, \alpha_{0}=0.2, \alpha_{1}=9.0$ and $\alpha_{2}=5.0, X_{1}$ is the gene labeled as Msa.1.0, $X_{268}$ is the gene labeled as Msa.11254.0. The average missing proportions among 500 replications corresponding to the above presented missingness data mechanism is about $48.5 \%$.

For this artificially created missing dataset, the above proposed DCNI-SIS procedure is utilized to identify the significant genes. For comparison,

we also consider the SIS (Fan and Lv, 2008), SIRS (Zhu et al., 2011), DC-SIS (Li, Zhong and Zhu, 2012), DF-SIS (Wu and Yin, 2015), MC-SIS (Wang et al., 2017), BMI procedures (e.g., MI-I, MI-S and V-D methods) (Wang and Li, 2018) and the CC method.

Similar to Experiment 1, we take the kernel function $K(\cdot)=\prod_{j=1}^{s_{n}^{\delta}} K_{j}(\cdot)$ as $K_{j}\left(z_{j}\right)=0.75\left(1-z_{j}^{2}\right)_{+}$, and set the bandwidth as $h_{j}=C_{j} \hat{\sigma}_{z_{j}} n^{-1 / 5}$, where $C_{j}=1$ and $\hat{\sigma}_{z_{j}}$ is the standard deviation of observations $\left\{Z_{i j}: i=1, \ldots, n ; j=1, \ldots, s_{n}^{\delta}\right\}$. For the given model size $d_{1}=\lfloor n / \log (n)\rfloor=8$ and $d_{2}=2\lfloor n / \log (n)\rfloor=16$, results for $C P_{1}, C P_{2}$ and $C P_{a}$ in identifying active predictors Msa.2134.0 and Msa.2877.0 are given in Table 5. Inspection of Table 5 indicates that the DCNI-SIS procedure outperforms other feature screening procedures in that the former has larger $\mathrm{CP}_{a}$ 
values than the latter.

\section{Conclusions}

In various missing data literature, it is common to artificially assume a parametric or semiparametric model with some pre-specified covariates for the considered missingness data mechanism. However, the plausibility of the posited model is doubtful. To address this issue, this paper investigates the feature screening problem for ultrahigh dimensional data in the presence of missing responses at random. A new feature screening procedure is proposed to simultaneously select important predictors associated with response variable and significant covariates associated with missing indicators by utilizing the nonparametric conditional mean imputation technique and the distance correlation and mean-variance indexes measuring the dependence between two random variables. A modified iterative algorithm is developed to find the optimal tuning parameter, which works well for missingness data mechanism model. The proposed feature screening procedure has the following merits. First, it is model-free because it dose not require assuming a regression model for the relationship between response and predictors, and a missingness data mechanism model for missing indicator. Second, it can be used to screen the nonlinear relationships between response and predictors, and missing indictor and covariates. Third, it is computationally feasible. Fourth, it is robust to the misspecification of propensity score functions. 
Under some regularity conditions, we show the sure screening property of the proposed screening procedure. Several simulation studies are conducted to investigate the finite sample performance of the proposed screening procedure. Empirical results evidence that the proposed screening procedure works well even if missingness data mechanism model is misspecified and the proportion is high, and is robust to heavy-tailed distributions for response. An example from the cardiomyopathy microarray dataset is illustrate by the proposed screening procedure.

Although we consider the case that response is subject to missing at random, the proposed feature screening procedure can be extended to missing not at random for response and/or covariates, which is quite challenging because the propensity score function depends on missing response. Also, we did not consider the asymptotic distribution of $\hat{\omega}_{k}$, which is rather challenging due to the complicated analytic expression of $\hat{\omega}_{k}$. We are working on these topics.

\section{Supplementary Materials}

Supplementary Materials available in the attached file include technical proofs and tables.

\section{Acknowledgements}

The authors are grateful to the Editor, an Associate Editor and two anonymous referees for their constructive suggestions that greatly improved this manuscript. 
This work was supported by the grant from the Key Project of the National Natural Science Foundation of China (Grant No.: 11731011).

\section{References}

Bouř, P., Kus, V. and Franc, J. (2017). Kernel and divergence techniques in high energy physics separations. Journal of Physics: Conference Series 898, pp. 072004.

Chang, J., Tang, C. and Wu, Y. (2013). Local independence feature screening for nonparametric and semiparametric models by marginal empirical likelihood. The Annals of Statistics 44, pp. 515-539.

Chen, X., Wan, A.T.K. and Zhou, Y. (2015). Efficient Quantile Regression Analysis With Missing Observations. Journal of the American Statistical Association 110, pp. 723-741.

Cheng, P. E. and Chu, C. K. (1996). Kernel estimation of distribution functions and quantiles with missing data. Statistica Sinica 6, pp. 63-78.

Cui, H., Li, R. and Zhong, W. (2015). Model-free feature screening for ultrahigh dimensional discriminant analysis. Journal of the American Statistical Association 110, pp. 630-641.

Fan, J., Feng, Y. and Song, R. (2011). Nonparametric independence screening in sparse ultra-highdimensional additive models. Journal of the American Statistical Association 106, pp. 544-557.

Fan, J. and Li, R. (2001). Variable selection via nonconcave penalized likelihood and its oracle properties. Journal of the American Statistical Association 96, pp. 1348-1360.

Fan, J. and Lv, J. (2008). Sure independence screening for ultrahigh dimensional feature space. J. Royal. Stat. Soc. Ser. B 70, pp. 849-911. 
Fan, J., Samworth, R. and Wu, Y. (2009). Ultrahigh dimensional feature selection: beyond the linear model. Journal of Machine Learning Research 10, pp. 1829-1853.

Fan, J. and Song, R. (2010). Sure independence screening in generalized linear models with NPdimensionality. The Annals of Statistics 38, pp. 3567-3604.

Huang, D., Li, R. and Wang, H. (2014). Feature screening for ultrahigh dimensional categorical data with applications. Journal of Business and Economic Statistics 32, pp. 237-244.

Lai, P., Liu, Y., Liu, Z. and Wan, Y. (2017). Model free feature screening for ultrahigh dimensional data with responses missing at random. Computational Statistics and Data Analysis 105, pp. 201-216.

Li, G., Peng, H., Zhang, J. and Zhu, L. (2012). Robust rank correlation based screening. The Annals of Statistics 40, pp. 1846-1877.

Li, R., Zhong, W. and Zhu, L. (2012). Feature screening via distance correlation learning. Journal of the American Statistical Association 107, pp. 1129-1139.

Little, R. J. A. and Rubin, D. B. (2002). Statistical Analysis with Missing Data (2 ed.). New York: Wiley.

Liu, J., Li, R. and Wu, R.(2014). Feature selection for varying coefficient models with ultrahighdimensional covariates. Journal of the American Statistical Association 109, pp. 266-274.

Mai, Q. and Zou, H. (2015). The fused Kolmogorov filter: a nonparametric model-free screening method. The Annals of Statistics 43, pp. 1471-1497.

Ni, L. and Fang, F. (2016). Entropy-based model-free feature screening for ultrahigh-dimensional multi- 
class classification. J. of Nonparametric Statistics 28, pp. 515-530.

Shao, L., Yu, Y. and Zhou, Y. (2016). Sure feature screening for high-dimensional dichotomous classification. Science China: Mathematics 59, pp.2527-2542.

Tang, N., Xia, L. and Yan, X. (2019). Feature screening in ultrahigh-dimensional partially linear models with missing responeses at random. Computational Statistics and Data Analysis 133, pp. 208-227.

Tang, N., Zhao, P. and Zhu, H. (2014). Empirical likelihood for estimating equations with nonignorably missing data. Statistica Sinica 24, pp. 723-747.

Tibshirani, R. (1996). Regression shrinkage and selection via the lasso. J. R. Stat. Soc. Ser. B 58, pp. $267-288$

Wang, L., Liu, J., Li, Y. and Li, R. (2017). Model-free conditional independence feature screening for ultrahigh dimensional data. Science China: Mathematics 60, pp. 551-568.

Wang, Q. and Li, Y. (2018). How to make model-free feature screening approaches for full data applicable to the case of missing response. Scandinavian Journal of Statistics 45, pp. 324-346.

Wu, Y. and Yin, G. (2015). Conditional quantile screening in ultrahigh-dimensional heterogeneous data. Biometrika 102, pp. 65-76.

Yan, X., Tang, N., Xie, J., Ding, X. and Wang, Z. (2018). Fused meanCvariance filter for feature screening. Computational Statistics and Data Analysis 122, pp. 18-32.

Zhang, C. (2010). Nearly unbiased variable selection under minimax concave penalty. The Annals of Statistics 38, pp. 894-942. 
Zhong, W., Zhu, L., Li, R. and Cui, H. (2016). Regularized quantile regression and robust feature screening for single index models. Statistica Sinica 26, pp. 69-95.

Zhou, Y., Wan, A. T. K., and Wang, X. (2008). Estimating Equation Inference with Missing Data. Journal of the American Statistical Association 103, pp.1187-1199.

Zhu, L. P., Li, L., Li, R. and Zhu, L. X. (2011). Model-free feature screening for ultrahigh dimensional data. Journal of the American Statistical Association 106, pp. 1464-1475.

Zhu, L. X. and Fang, K. T. (1996). Asymptotics for kernel estimate of sliced inverse regression. The Annals of Statistics 24, pp.1053-1068.

Zou, H. (2006). The adaptive lasso and its oracle properties. Journal of the American Statistical Association 101, pp. 1418-1429.

Yunnan Key Laboratory of Statistical Modeling and Data Analysis, Yunnan University, Kunming 650500,

China

E-mail: linli_xia1@163.com

Yunnan Key Laboratory of Statistical Modeling and Data Analysis, Yunnan University, Kunming 650500,

China

E-mail: nstang@ynu.edu.cn 
REFERENCES

Table 1. Performance of MV-SIS procedure for screening covariates in propensity score function in Experiment 1.

\begin{tabular}{|c|c|c|c|c|c|c|c|c|c|c|c|c|c|}
\hline \multirow[b]{2}{*}{ Case } & \multirow[b]{2}{*}{ Method } & \multicolumn{4}{|c|}{$\varepsilon \sim N(0,1)$} & \multicolumn{4}{|c|}{$\varepsilon \sim t(3)$} & \multicolumn{4}{|c|}{$\varepsilon \sim \operatorname{Cauchy}(0,1)$} \\
\hline & & AMS & $\mathrm{CF}$ & OF & UF & AMS & $\mathrm{CF}$ & $\mathrm{OF}$ & UF & AMS & $\mathrm{CF}$ & OF & UF \\
\hline \multirow[t]{2}{*}{ (i) } & MV-TM & 1.002 & 0.998 & 0.002 & 0.000 & 1.008 & 0.992 & 0.008 & 0.000 & 1.004 & 0.996 & 0.004 & 0.000 \\
\hline & MV-T & 1.048 & 0.952 & 0.048 & 0.000 & 1.060 & 0.940 & 0.060 & 0.000 & 1.034 & 0.966 & 0.034 & 0.000 \\
\hline \multirow[t]{2}{*}{ (ii) } & MV-TM & 2.000 & 1.000 & 0.000 & 0.000 & 2.000 & 1.000 & 0.000 & 0.000 & 2.000 & 1.000 & 0.000 & 0.000 \\
\hline & MV-T & 2.004 & 0.996 & 0.004 & 0.000 & 2.002 & 0.998 & 0.002 & 0.000 & 2.006 & 0.994 & 0.006 & 0.000 \\
\hline \multirow[t]{2}{*}{ (iii) } & MV-TM & 1.010 & 0.992 & 0.008 & 0.000 & 1.010 & 0.990 & 0.010 & 0.000 & 1.010 & 0.990 & 0.010 & 0.000 \\
\hline & MV-T & 1.060 & 0.942 & 0.058 & 0.000 & 1.052 & 0.948 & 0.052 & 0.000 & 1.066 & 0.934 & 0.066 & 0.000 \\
\hline
\end{tabular}

Note: 'MV-TM' and 'MV-T' represent the MV-SIS approaches with $d_{\delta}$ determined by the mod-

ified tuning parameter selection method introduced in Section 2.2 and the algorithm introduced

in $\mathrm{Ni}$ and Fang (2016), respectively.

Table 2. Performance of the DCNI-SIS procedure in identifying active predictors in Experiment 1.

\begin{tabular}{|c|c|c|c|c|c|c|c|c|c|c|c|c|c|}
\hline \multirow[b]{2}{*}{ Case } & \multirow[b]{2}{*}{ Method } & \multicolumn{4}{|c|}{$\varepsilon \sim N(0,1)$} & \multicolumn{4}{|c|}{$\varepsilon \sim t(3)$} & \multicolumn{4}{|c|}{$\varepsilon \sim \operatorname{Cauchy}(0,1)$} \\
\hline & & AMS & $\mathrm{CF}$ & $\mathrm{OF}$ & UF & AMS & $\mathrm{CF}$ & $\mathrm{OF}$ & UF & AMS & $\mathrm{CF}$ & OF & UF \\
\hline \multirow[t]{2}{*}{ (i) } & DUN & 5.066 & 0.506 & 0.274 & 0.220 & 4.860 & 0.508 & 20 & 0.272 & 5.582 & 0.322 & .282 & 0.396 \\
\hline & DCNI-T & 5.312 & 0.438 & 0.352 & 0.210 & 5.258 & 0.450 & 0.306 & 0.244 & 5.952 & 0.270 & 0.372 & 0.358 \\
\hline \multirow[t]{2}{*}{ (ii) } & DCNI-TM & 4.526 & 0.704 & 0.186 & 0.110 & 4.514 & 0.718 & 0.164 & 0.118 & 4.844 & 0.508 & 0.228 & 0.264 \\
\hline & DCNI-T & 4.718 & 0.660 & 0.244 & 0.096 & 4.600 & 0.678 & 0.226 & 0.096 & 5.080 & 0.462 & 0.310 & 0.228 \\
\hline \multirow[t]{2}{*}{ (iii) } & DCNI-TM & 5.038 & 0.474 & 0.272 & 0.254 & 4.910 & 0.478 & 0.232 & 0.290 & 5.510 & 0.274 & 0.268 & 0.458 \\
\hline & DCNI-T & 5.362 & 0.426 & 0.328 & 0.246 & 5.126 & 0.428 & 0.308 & 0.264 & 6.064 & 0.236 & 0.350 & 0.414 \\
\hline
\end{tabular}

Note: 'DCNI-TM' and 'DCNI-T' represent the DCNI-SIS approaches with $d_{n}$ determined by

the modified tuning parameter selection method introduced in Section 2.2 and the algorithm introduced in Ni and Fang (2016), respectively. 
REFERENCES

Table 3. Performance of $\mathrm{CP}_{j}$ 's and $C P_{a}$ for various screening methods in identifying active predictors in

Experiment 1.

\begin{tabular}{|c|c|c|c|c|c|c|c|c|c|c|c|c|c|c|c|c|}
\hline \multirow[b]{2}{*}{ Case } & \multirow[b]{2}{*}{ Method } & \multicolumn{5}{|c|}{$\varepsilon \sim N(0,1)$} & \multicolumn{5}{|c|}{$\varepsilon \sim t(3)$} & \multicolumn{5}{|c|}{$\varepsilon \sim \operatorname{Cauchy}(0,1)$} \\
\hline & & $\mathrm{CP}_{1}$ & $\mathrm{CP}_{2}$ & $\mathrm{CP}_{3}$ & $\mathrm{CP}_{4}$ & $\mathrm{CP}_{a}$ & $\mathrm{CP}_{1}$ & $\mathrm{CP}_{2}$ & $\mathrm{CP}_{3}$ & $\mathrm{CP}_{4}$ & $\mathrm{CP}_{a}$ & $\mathrm{P}_{1}$ & $\mathrm{CP}_{2}$ & $\mathrm{CP}_{3}$ & $\mathrm{CP}_{4}$ & $\mathrm{CP}_{a}$ \\
\hline \multirow{10}{*}{ (i) } & MI-I & 0.994 & 0.996 & 1.000 & 0.882 & 0.874 & 0.992 & 0.998 & 1.000 & 0.904 & 0.896 & 0.952 & 0.998 & 1.000 & 0.786 & 0.748 \\
\hline & $\mathrm{N}$ & 0.044 & 0.998 & 000 & 0.040 & 0.002 & .036 & 0.996 & 1.000 & 0.020 & 0.000 & .030 & 0.994 & 1.0 & 0.034 & 0.000 \\
\hline & V-D & 0.996 & 0.818 & 0.940 & 0.884 & 0.718 & .992 & 0.788 & 0.944 & 0.906 & 0.690 & .952 & 0.660 & 0. & 0.780 & 0.470 \\
\hline & MC- & 1.000 & 0.924 & 0.982 & 0.990 & 0.902 & 1.000 & 0.910 & 0.978 & 0.988 & 0.876 & .000 & 0.840 & 0.958 & 0.958 & 0.770 \\
\hline & SIS & 0.648 & 0.362 & 0.538 & 0.474 & 0.122 & 0.660 & 302 & 0.498 & 0.468 & 0.092 & 0.452 & 0.232 & 0.3 & 0.350 & 0.048 \\
\hline & & 1 & 0.936 & 0.988 & 0. & 0.918 & 000 & 0.902 & 0.982 & 0.988 & 0.872 & 0.994 & 42 & 0. & 0.950 & 0.776 \\
\hline & $\mathrm{D}$ & 0.870 & 0.264 & 0.510 & 0.578 & 0.088 & 0.908 & 0.220 & 0.440 & 0.550 & 0.058 & 0.684 & 0.194 & 0.32 & 0.420 & 0.030 \\
\hline & & 1 & 818 & 0.940 & & 6 & & 786 & & & & & & & & 0.520 \\
\hline & $\mathrm{DCN}$ & 1.000 & 0.990 & 1.000 & 0.980 & 0.970 & 1.000 & 0.990 & 1.000 & 0.974 & 0.964 & 0.990 & 0.990 & 1.000 & 0.918 & 0.902 \\
\hline & & 1.000 & 0.894 & 0.972 & 0.980 & 0.858 & .000 & 0.856 & 0.968 & 0.982 & 0.818 & & 90 & & 0.932 & 0.686 \\
\hline \multirow{8}{*}{ (ii) } & MI-I & 0.998 & 1.000 & 1.000 & 0.948 & 0.946 & 0.998 & 1.000 & 1.000 & 0.948 & 0.946 & 0.966 & 1.000 & 1.000 & 0.876 & 0.848 \\
\hline & & 2 & 1.000 & 1.000 & 0.032 & 0.000 & 0.022 & 1.000 & 1.0 & 0.032 & 0.000 & 038 & & & 34 & 0.002 \\
\hline & V-D & 0.998 & 0.722 & 0.988 & 0.950 & 0.686 & 0.998 & 0.670 & 0.964 & 0.948 & 0.614 & 0.966 & 0.522 & 0.936 & 0.876 & 0.438 \\
\hline & MC-SIS & 1.000 & 0.852 & 0.994 & 1.000 & 0.848 & 1.000 & 0.792 & 0.992 & 0.996 & 0.780 & 1.000 & 0.706 & 0. & 0.988 & 0.680 \\
\hline & SIS & 0.664 & 0.334 & 0.538 & 0.502 & 0.094 & 0.686 & 0.290 & 0.544 & 0.522 & 0.088 & 0.472 & 0.214 & 0.3 & 0.374 & 0.048 \\
\hline & $\mathrm{RS}$ & 1.000 & 0.878 & 0.998 & 0.998 & 0.876 & 1.000 & 0.852 & 0.994 & 0.998 & 0.844 & 1.000 & 0.780 & 0.980 & 0.992 & 0.760 \\
\hline & & 0.896 & 0.168 & 0.550 & 0.5 & 0.0 & 0.908 & 0.144 & 0. & 0.628 & 0.048 & 0.740 & 0.112 & & 0.482 & 0.028 \\
\hline & $\mathrm{D}$ & 0.998 & 0.722 & 0.988 & 0.976 & 0.698 & 1.000 & 0.670 & 0.964 & 0.992 & 0.65 & 0.99 & 0.522 & 0.936 & 0.930 & 0.470 \\
\hline \multirow{12}{*}{ (iii) } & & 1.0 & 1.000 & 0 & & & & 1.000 & & & & 1.000 & 1.000 & & 0.966 & 0.966 \\
\hline & $\mathrm{CC}$ & 1.000 & 0.792 & 0.992 & 0.994 & 0.780 & 1.000 & 0.736 & 0.986 & 0.996 & 0.722 & 1.000 & 0.638 & 0.958 & 0.976 & 0.602 \\
\hline & & 0.986 & 0.996 & 1.000 & 0.852 & 0.836 & 0.9 & 0.998 & 1.000 & 0.868 & 0.858 & 0.936 & 0.994 & 1. & 0.722 & 0.680 \\
\hline & MI-S & 0.036 & 0.998 & 1.000 & 0.026 & 0.000 & 0.032 & 0.996 & 1.0 & 0.020 & 0.002 & 0.044 & 0.99 & 1. & 0.042 & 0.000 \\
\hline & $V-D$ & 0.986 & 0.758 & 0.904 & 0.856 & 0.622 & 0.988 & 0.732 & 0.922 & 0.872 & 0.594 & 0.936 & 0.604 & 0.778 & 0.718 & 0.380 \\
\hline & MC-SIS & 1.000 & 0.914 & 0.978 & 0.982 & 0.882 & 1.000 & 0.882 & 0.970 & 0.986 & 0.842 & 1.000 & 0.800 & 0.912 & 0.964 & 0.728 \\
\hline & SIS & 0.570 & 0.280 & 0.396 & 0.418 & 0.060 & 0.584 & 0.246 & 0.360 & 0.430 & 0.052 & 0.406 & 0.194 & 0.238 & 0.298 & 0.026 \\
\hline & IRS & 1.000 & 0.916 & 0.980 & 0.982 & 0.886 & 1.000 & 0.894 & 0.978 & 0.986 & 0.862 & 1.000 & 0.830 & 0.930 & 0.960 & 0.760 \\
\hline & DF-SIS & 0.832 & 0.212 & 0.384 & 0.524 & 0.044 & 0.858 & 0.178 & 0.318 & 0.510 & 0.048 & 0.642 & 0.168 & 0.222 & 0.330 & 0.010 \\
\hline & DC-SIS & 0.996 & 0.758 & 0.904 & 0.934 & 0.674 & 1.000 & 0.732 & 0.922 & 0.958 & 0.668 & 0.976 & 0.604 & 0.778 & 0.836 & 0.454 \\
\hline & DCNI-SIS & 1.000 & 0.988 & 1.000 & 0.960 & 0.950 & 1.000 & 0.992 & 1.000 & 0.970 & 0.962 & 0.996 & 0.994 & 1.000 & 0.920 & 0.910 \\
\hline & $\mathrm{CC}$ & 1.000 & 0.866 & 0.960 & 0.970 & 0.816 & 1.000 & 0.834 & 0.944 & 0.980 & 0.774 & 0.998 & 0.764 & 0.876 & 0.952 & 0.672 \\
\hline
\end{tabular}


REFERENCES

Table 4 . The 5\%, 25\%, 50\%, 75\% and $95 \%$ quantiles of MMS for various screening methods in identifying active predictors in Experiment 1.

\begin{tabular}{|c|c|c|c|c|c|c|c|c|c|c|c|c|c|c|c|c|}
\hline \multirow[b]{2}{*}{ Case } & \multirow[b]{2}{*}{ Method } & \multicolumn{5}{|c|}{$\varepsilon \sim N(0,1)$} & \multicolumn{5}{|c|}{$\varepsilon \sim t(3)$} & \multicolumn{4}{|c|}{$\varepsilon \sim \operatorname{Cauchy}(0,1)$} & \multirow[b]{2}{*}{$95 \%$} \\
\hline & & $5 \%$ & $25 \%$ & $50 \%$ & $75 \%$ & $95 \%$ & $5 \%$ & $25 \%$ & $50 \%$ & $75 \%$ & $95 \%$ & $5 \%$ & $25 \%$ & $50 \%$ & $75 \%$ & \\
\hline \multirow{10}{*}{ (1) } & MI-I & 4 & 4 & 6 & 17 & 101.4 & 4 & 4 & 6 & 14 & 73 & 4 & 5 & 10 & 38 & 177.1 \\
\hline & MI-S & 219.0 & 495.5 & 694.5 & 872.3 & 978 & 57.9 & 500.3 & 698.5 & 864.3 & 975.2 & 252.0 & 493.8 & 728.5 & 874.3 & 971 \\
\hline & V-D & - & - & - & - & - & - & - & - & - & - & - & - & - & - & - \\
\hline & MC-SIS & 4 & 4 & 5 & 13 & 78.1 & 4 & 4 & 6 & 15 & 77.2 & 4 & 5 & 10 & 32.3 & 165.2 \\
\hline & SIS & 15 & 93.8 & 272 & 630.3 & 904.6 & 21 & 113 & 284.5 & 625.8 & 905.2 & 42.0 & 230.5 & 521.5 & 776 & 958.1 \\
\hline & SIRS & 4 & 4 & 5 & 11.3 & 71 & 4 & 4 & 5 & 14.3 & 88.1 & 4 & 5 & 10 & 32 & 177.1 \\
\hline & DF-SIS & 23.0 & 125 & 324.5 & 613.3 & 917 & 26.9 & 175.5 & 366.5 & 644.8 & 914.1 & 55.0 & 244.5 & 482 & 748.3 & 949.1 \\
\hline & DC-SIS & 4 & 5 & 11 & 36.3 & 203.5 & 4 & 5 & 13 & 40 & 188.2 & 4 & 10 & 34 & 96 & 427.3 \\
\hline & DCNI-SIS & 4 & 4 & 4 & 5 & 26 & 4 & 4 & 4 & 5.3 & 26.1 & 4 & 4 & 5 & 9.3 & 69.1 \\
\hline & $\mathrm{CC}$ & 4 & 4 & 6 & 19 & 126.5 & 4 & 4 & 7 & 21 & 125.2 & 4 & 6 & 16 & 48.3 & 234.1 \\
\hline \multirow{10}{*}{ (ii) } & MI-I & 4 & 4 & 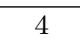 & 8 & 39.1 & 4 & 4 & 4 & 0 & 39 & 4 & 4 & 6 & 18 & 95.1 \\
\hline & MI-S & 186.5 & 495.8 & 701.5 & 860 & 976 & 236.9 & 510.5 & 703 & 863.3 & 974.1 & 221.8 & 505.5 & 684.5 & 874.5 & 974 \\
\hline & V-D & - & - & - & - & - & - & - & - & - & - & - & - & - & - & - \\
\hline & MC-SIS & 4 & 4 & 7 & 18 & 148.1 & 4 & 4 & 8 & 31 & 175.1 & 4 & 6 & 15 & 49 & 281 \\
\hline & IS & 22.9 & 107.8 & 293.5 & 595 & 915.3 & 23 & 114 & 288.5 & 584.3 & 892.2 & 40.0 & 232.3 & 517.5 & 796.3 & 963.2 \\
\hline & SIRS & 4 & 4 & 5 & 15 & 94.2 & 4 & 4 & 6 & 18 & 149.3 & 4 & 5 & 10 & 35 & 241.3 \\
\hline & -SIS & 34.9 & 167.3 & 372 & 640 & 926.1 & 41.0 & 201 & 415 & 684.5 & 941.2 & 68.0 & 264.5 & 489 & 749.3 & 934.1 \\
\hline & DC-SIS & 4 & 5.8 & 14 & 54 & 289 & 4 & 6 & 17 & 70.3 & 328.1 & 4 & 12 & 41.5 & 142.3 & 550.3 \\
\hline & DCNI-SIS & 4 & 4 & 4 & 4 & 9 & 4 & 4 & $x$ & 4 & 11 & 4 & 4 & 4 & 6 & 24.2 \\
\hline & $\mathrm{CC}$ & 4 & 5 & 9 & 33 & 220.8 & 4 & 5 & 11 & 45 & 240.2 & 4 & 7 & 21.5 & 86 & 390.1 \\
\hline \multirow{10}{*}{ (iii) } & MI-I & 4 & 4 & 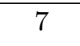 & 21.3 & 135.1 & 4 & 4 & 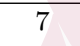 & 18 & 80.2 & 4 & 6 & 15 & 53 & 191.1 \\
\hline & MI-S & 14.8 & 481 & 705 & 870.8 & 977 & 23.0 & 513.5 & 722 & 869 & 976.1 & 249.9 & 478.8 & 684 & 853.5 & 974.1 \\
\hline & V-D & - & - & - & - & - & - & - & - & - & - & - & - & - & - & - \\
\hline & MC-SIs & 4 & 4 & 6 & 15 & 100.8 & 4 & 4 & 7 & 21 & 108.2 & 4 & 6 & 12 & 42 & 206.3 \\
\hline & SIS & 31.9 & 154 & 390 & 703.3 & 940 & 36.0 & 187 & 409 & 661.5 & 931.4 & 73 & 346.3 & 605.5 & 814 & 972.1 \\
\hline & $\mathrm{BS}$ & 4 & 4 & 6 & 15 & 108 & 4 & 4 & 6 & 18 & 121.1 & 4 & 5 & 12 & 36 & 238 \\
\hline & DF-SIS & 45.0 & 198.5 & 414.5 & 714.3 & 947.1 & 41.0 & 239.3 & 467.5 & 740 & 946.2 & 95.7 & 349 & 596 & 838.5 & 974.2 \\
\hline & DC-SIS & 4 & 6 & 16 & 57 & 243.1 & 4 & 7 & 18.5 & 57.3 & 237.2 & 5 & 16 & 45 & 144.8 & 397.1 \\
\hline & DCNI-SIS & 4 & 4 & 4 & 6 & 35.2 & 4 & 4 & 4 & 6 & 25.1 & 4 & 4 & 5 & 12 & 59.2 \\
\hline & $\mathrm{CC}$ & 4 & 4 & 8 & 23.3 & 157.3 & 4 & 5 & 9 & 32 & 153.1 & 4 & 7 & 19 & 63.5 & 344.5 \\
\hline
\end{tabular}

Table 5. The selected predictors among 500 repetitions in the real example.

\begin{tabular}{cccccccc}
\hline & \multicolumn{3}{c}{$d_{1}=8$} & & \multicolumn{3}{c}{$d_{2}=16$} \\
\cline { 2 - 3 } Method & Msa.2134.0 & Msa.2877.0 & $\mathrm{CP}_{a}$ & & Msa.2134.0 & Msa.2877.0 & $\mathrm{CP}_{a}$ \\
\hline MI-I & 0.672 & 0.588 & 0.588 & & 0.674 & 0.682 & 0.644 \\
MI-S & 0.000 & 0.000 & 0.000 & & 0.000 & 0.000 & 0.000 \\
V-D & 0.672 & 0.588 & 0.588 & & 0.674 & 0.682 & 0.644 \\
MC-SIS & 0.118 & 0.966 & 0.100 & & 0.200 & 1.000 & 0.200 \\
SIS & 0.012 & 1.000 & 0.012 & & 0.064 & 1.000 & 0.064 \\
SIRS & 0.474 & 1.000 & 0.474 & & 0.734 & 1.000 & 0.734 \\
DF-SIS & 0.000 & 0.000 & 0.000 & & 0.000 & 0.002 & 0.000 \\
DC-SIS & 0.356 & 0.684 & 0.244 & & 0.722 & 0.974 & 0.706 \\
DCNI-SIS & 0.810 & 0.876 & 0.690 & & 0.950 & 0.964 & 0.914 \\
CC & 0.040 & 0.812 & 0.030 & & 0.070 & 0.938 & 0.070 \\
\hline
\end{tabular}

